\title{
MAGNETOSENSITIVE CONDUCTIVITY OF ALUMINUM AND THE ADVANTAGE OF CORBINO GEOMETRY
}

\author{
B.B.Boiko, V.R.Sobol, O.N.Mazurenko, and A.A. Drozd \\ Institute of Physics of Solids and Semiconductors \\ Minsk, 220072, Belarus
}

\section{ABSTRACT}

The problem of low temperature high density charge transport in disk shaped sample (Corbino geometry) has been investigated experimentally and analytically for polycrystalline aluminum having residual resistance ratio of $10^{4}$. The values of magnetoresistance and self magnetic field stimulated by Hall drift in an external magnetic field up to $8 \mathrm{~T}$ has been estimated for exciting current of the order $10^{3} \mathrm{~A}$ flowing through the disk samples. The analysis of observed data has been done on the base of equations for connection of current density with electric and magnetic field, the relaxation processes having been taken into account. Definite correlations between experiment and theory for disk shaped sample being inductance were established. These are: magnetoresistance, self magnetic field, its distribution and energy, characteristic transition time. Some possible variants of application of studied phenomena for energy storage and transmission. control systems of cryogenic electric circuits are proposed with account of characteristic inductive and resistive properties of sample organized in such manner.

\section{INTRODUCTION}

Aluminum being uncompensated metal is a perspective material used in equipment of cryogenic electrotransmission and magnetic field generation where there need high current windings. Due to low magnetoresistance it has a definite advantages over other conductors. On the other hand the magnetoconductivity of Al has a strong dependence on a magnetic field. In cryogenic range of temperature it is rather difficult to realize the regime of voltage supply and at first a high magnetosusceptibility of charge transport is unlikely to be achieved however it is possible in Corbino geometry. This geometry as a method of organization of a sample form is used in the technique of low temperature experiment to ensure the case of unlimited in transverse direction sample. It gives possibility to exclude the Hall field affect to the electron movement and the feature 
connected with it. So the peculiarities of electron scattering can be studied by means of measuring the reversal diagonal components of electro- and heat conductivity tensor ${ }^{1-7}$.

In this paper the complex experimental and analytical study of high density charge transport in aluminum specimen in Corbino geometry has been done. The sample being regarded as an opened for energy exchange systems. The peculiarities of such charge transport at different values of current were studied with the aim of seeking the ways of application of this geometry not only for fundamental research but for solving of applied problems as well.

\section{EXPERIMENTAL TECHNIQUE}

The specimens for investigation were disk-shaped samples of some sizes: the inner and outer diameters of 5 and $35 \mathrm{~mm}$ respectively, the thickness $t$ being of $2 \mathrm{~mm}$ (fig.1). The residual resistance ratio of material was of $10^{4}$. When fabricated the samples were annealed at $400 \mathrm{~K}$ during 24 hours. The investigations were made in current supply regime, the maximal magnitude of current through the samples being $10^{3} \mathrm{~A}$. An external magnetic field was up to $8 \mathrm{~T}$. For temperature measurements the samples were placed into thermostat with automatic monitoring of temperature. Heat resistance converters and thermocouples were used as temperature sensors. During other measurements the samples were placed in the medium of liquid helium so the heat removal being the boiling in large volume. The registration method consisted of determination of integral magnetoconductivity, voltage-current characteristics, distribution of the ou'n magnetic field along radius as a difference between a total magnetic field and external one.

\section{EXPERIMENTAL RESULTS AND DISCUSSION}

\section{Magnetosusceptibility of conductivity and the relaxation processes}

High level of resistivity of uncompensated metal samples in Corbino geometry is known to be ensured by the conditions of strong magnetic field, i.e. $I / r=\omega r \gg>1$, here $l$ - electron free length, $r$ - Larmor radius, $\omega$ - cyclotron frequency, $\tau$ - characteristic time. Here we are regarding the degree of affect of magnetic field to the electron kinetic and relaxation processes. The resistance as a function of magnetic field at different temperatures is presented in fig.2. Characteristically that the experimental behaviour is not entirely quadratic however the law of dependence on magnetic field is almost the same for different temperature. Respectively one can claim that the dirty limit is not realized in this geometry even at temperature of liquid helium. Actually magne toresistance evaluated with a help of value of resistance in zero magnetic field is greater of experimentally observed one. This discreapancy is enlarged with magnetic field magnification. Thus the actual relaxation time is greater of calculated one and depends on $B$. The reason of this are the processes of small angle scattering with

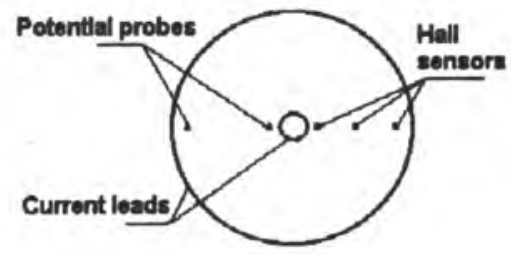

Figare 1. Disk shaped sample and its electric power supply. 


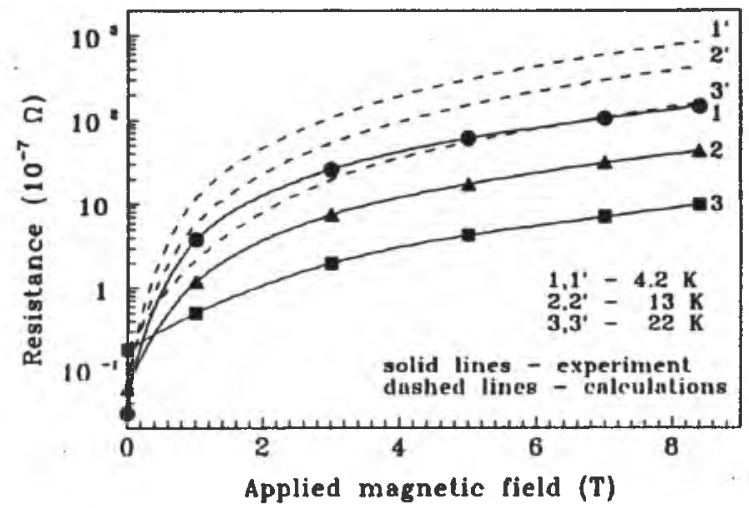

Figure 2. Sample resistance in an external magnetic field at different temperaturus.

respective umclapp and magnetic breakdown. The conductivity itself is determined by the summing of different groups of electron and whereas for most of them the conductivity is proportional $B^{-2}$ for a small part of them being placed near Bragg planes the conductivity is proportional to $B^{0}$. Respectively integral conductivity is some larger than the estimation having made for electrons belonged only to closed orbits. So the magnetoresistance as a reversal value of conductivity shows less both a value and field dependence. Consequently the approximation of relaxation time describing acts of electron scattering on impurity atoms when a scattering probability is independent on electron wave vector and magnetic field magnitude is not adequate for this situation. Thus the presence in collision integral of modified transport coefficient must not be described by the usual term of relaxation time at least for this geometry. However this term is used in our experimental study of relaxation processes being the new characteristics describing all this complicated processes of macroscopical averaging over isoenergetical surface. In other words we shall use the term of relaxation time as a symbol of presentation of new phenomena by means of old convenient term.

\section{Self maguetism of electrons}

The boundary conditions in this case are realized for equipotential azimuthal contours, the Hall drift taking place. Respectively material coupling equation can be presented as follow phenomenological relation

$$
j=\sigma E+[j \times \omega \tau] ; \omega=\frac{e B}{m c}
$$

here $j$ - current density, $\sigma$ - conductivity, $E$ - electric field, $e$ and $m$ - electron charge and mass, $c$ - light speed.

Naturally the azimuthal motion of carriers besides of excessive resistivity ensures an appearance of self field component being coaxial to an external magnetic field. The self magnetic field may be much greater of the one in traditional geometry. This field is inhomogeneous along the radius and summarized algebraically with an external one acting to the motion of electrons as well. Following the expression for current value $j=n e v$, where $v$ is the velosity of drift, $n$ is the concentration of electrons, it is clear that the velosity of Hall drift is great many of collision drift and an electron path in 
transverse direction is $\infty \tau$ as much as the one in transport radial direction. There takes place spiral electron movement aroud inner contact. Such sample can be considered as inductance the number of winds being of the order of $\omega \tau$ because $j_{0}=\omega \tau j$, where $j_{0}$ is azimuthal current density. On the other hand the sample may be represented as single wind coil, the current of value of $₫ \tau I$ being made to flow through it.

The distribution of self magnetic field is presented in fig.3. Here are both the experimental data based on the measurement of normal to the sample surface component of the self field and the data calculated in effective relaxation time approximation. Notice that the self magnetic field coincides in direction with an external one when current flows to the sample center, there taking place paramagnetic regime of disk operation out of an external field direction. When a current flows out of the center the self and an external fields are antiparallel.

The end surface distribution of self magnetic field $B$ along the disk radius has been estimated with help of expression:

$$
B(R)=\frac{1}{c} \iiint \frac{j_{\theta}\left(R^{\prime}\right)\left(R-R^{\prime}\right)}{\left|R-R^{\prime}\right|^{3}} d V
$$

here $R$ - a radius vector of a seeking point, $R^{\prime}$ - a radius vector of current element having the density $j_{\theta}\left(R^{\prime}\right)$.

The calculation has been done for the approximation of limit case $t<R$ with help of complex variable function for summing on azimuth:

$$
B(R)=\frac{I_{\theta}}{c}\left\{-\frac{1}{R} \ln \left|\frac{R\left(R+\sqrt{R_{2}^{2}+R^{2}}\right.}{R_{2}\left(R+\sqrt{2 R^{2}}\right.}\right|+\frac{1}{2 R} \ln \left|\frac{\left(\sqrt{R_{2}^{2}+R^{2}}-R\right)\left(\sqrt{2 R^{2}}+R\right)}{\left(\sqrt{R_{2}^{2}+R^{2}}+R\right)\left(\sqrt{2 R^{2}}-R\right)}\right|+\frac{1}{\sqrt{R^{2}+R_{2}^{2}}}-\frac{1}{\sqrt{2 R^{2}}}\right\}
$$

Notice that the character of the observed self field well respects to the theoretical modified logarithmic dependence. Take into account that for samples with thickness of $2-4 \mathrm{~mm}$ the axial component of the self magnetic field measured on the end surface is of the order of magnitude with the intravolume field value. It is easy to define the spatially averaged density of energy of own magnetic field $W$ (fig.4). There is almost linear dependence of $W$ on value of current squared $\boldsymbol{P}$. The density of self magnetic

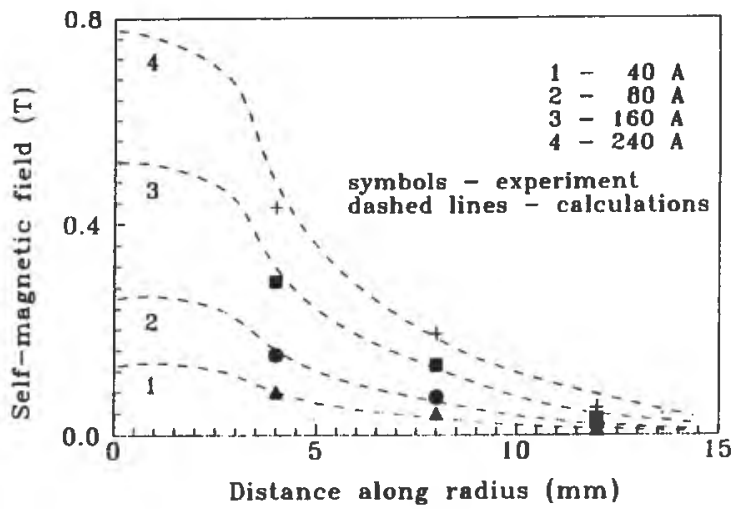

Fgure 3. Self magnetic field distribution along radius (end surface) for different currents. 


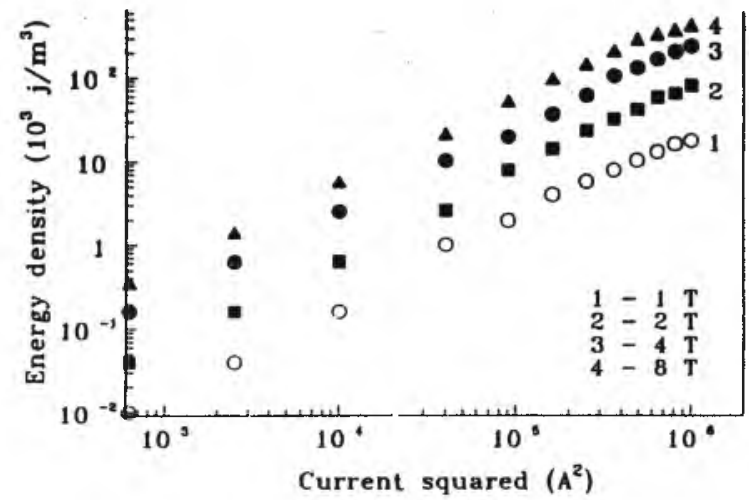

Figure 4. Averaged energy density of self magnetic field at different applied fields.

energy accumulated in the disk is of the same order of magnitude with traditional inductive accumulators and is much greater of that of capacitance one. This takes place when a magnitude of external magnetic field is of the order of $8 \mathrm{~T}$ and current value is of $10^{3} \mathrm{~A}$. This energy density may be realized at less external fields when a current is respectively increased.

\section{Some relations for disk shaped sample}

The observed data permit to evaluate some parameters of such sample being placed into an external magnetic field, current being made to flow between inner and outer contacts. First of all consider the typical relation of any inductance that is the ratio of its inductance to its resistance determining a characteristic time of transient process. Thus for samples being measured here the extreme magnitude of accumulated energy was of the order of $1 \mathrm{j}$ in sample volume $V$ of $1-2 \mathrm{~cm}^{3}$. The value of $L / R_{B} \sim W V / T^{2} R_{B}$ estimated both from experimental data and analytically in accordance with data in fig. 2 is presented in fig.5. So for the magnetic field $B=8 \mathrm{~T}$ and current $I \sim 10^{3} \mathrm{~A}$ the value $L / R_{B}$ is of the order of $1 \mathrm{sec}$. Notice that for other magnitudes of current and magnetic field there takes place the different order of this parameter being a rather small for such high level of energy density storage and for this case is ensured by high value of parameter $\omega \tau$ when the energy storage is due to azimutal current component.

On the basis of obtained data one can state that there are definite possibilities of applying this phenomena in cryogenic systems of energy storage and control circuits.

Thus disk or cylinder shaped element integrated with an external magnetic field of inductance energy storage, a pattern being a core and placing whithin that can enlarge the energy storage. This is possible providing all the volume of inductive accumulator (for example torroidal coil) is occupied by Corbino shaped aluminum specimen. Notice that the connection in serious is more desirable for increasing of energy storage because the same current flows through the coil and the sample. For the connection in parallel the current is inversely as an active resistance of coil and sample so the energy will be less. This concerns both current and voltage supply and for second mode the regime will be defined by a value of common resistance of this joint device.

It should be mentioned that such element integrated with an external magnetic field and supplied with independent power source may be inductive energy storage 


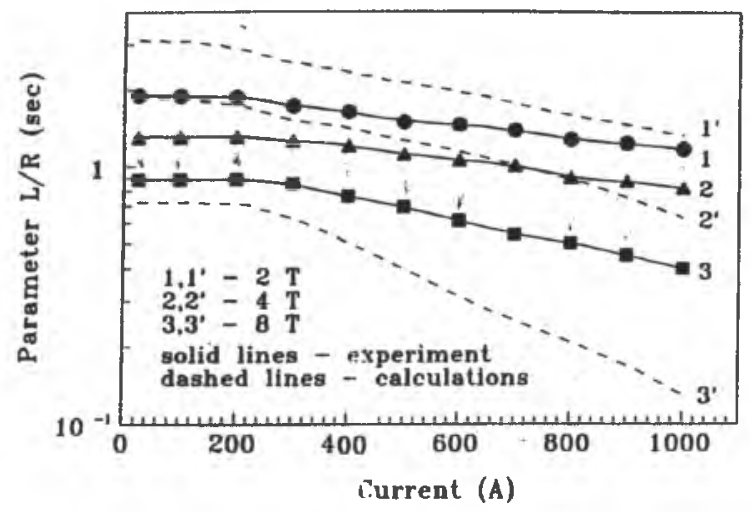

Figare 5. Characteristic transient time of a sample as an inductance.

itself. Its characteristic charge and discharge time will be defined by geometry, resistivity and levels of an external magnetic field and current supply. The high level of magnetoresistive effect (of the order of $10^{4}$ ) may be used for control cryogenic electrical circuits and devices having own strong magnetic field too. For instance in control system of charging and discharging circuits of inductive energy storage.

There are other possibilities of using these phenomena in cryogenic systems of control of circuits. Thus a disc shaped core joint with inductor in serious or in parallel will have a varying active and reactive resistance depending both on external magnetic field and on own magnetic field, the value of current and its direction. That is such joint device must be a parametrical inductor. Due to high magnetoresistive effect it may operate as both the current supply and voltage one. For example for our case the power supply with output resistance of $10^{-2} \Omega$ will be current supply when current is small, or magnetic field is zero, and voltage supply when current is high and magnetic field is of the order of $8 \mathrm{~T}$.

The susceptibility to the current direction means that acting magnetic field may be changed up to $20 \%$ for dia- and paramagnetic regime. Thus the total effective parameter $\omega \tau$ will have magnitude $100 \pm 10$ in this case (when current is of the order of $200 \mathrm{~A}$, an external magnetic field of $6 \mathrm{~T}$ ). So the inductance and active resistance differ for this case in direct proportion to square of the value $\omega \tau$, that is about $40 \%$ of mean significance.

So far we regarded only steady in time regime of operation. It should be mentioned that strong skin effect for material of such high level of conductivity leads to the nonstationary field penetrates into a small deepth. For example for a field having frequency $1 \mathrm{c} / \mathrm{s}$ the skin deepth is of the order of $0.1 \mathrm{~cm}$ both for normal and anomal skin effect approximation. So for the such and more higher frequency range different external alien field will slighly influence to the parameters of disk as a controlling element. High conductivity of material and skin effect will also complicate the processes of charging and discharging of disk sample as an inductor. So the transient processes will be determined not only with stationary parameters of sample but other characteristics such as geometry, frequency range, regime of supply as well as total properties of cryogenic electric circuits.

It shoud be mentioned that with help of experimental and theoretical data on magnetoresistance (fig.2) it is possible to estimate all necessary parameters and characteristics of disk shaped inductive element in temperature range up to liquid 
hydrogen. So the value of resistance enables to calculate the effective characteristic relaxation time and all other parameters. For example the accumulated energy density at hydrogen temperature will be a third of that at helium. The advantages of pure aluminum used in such manner may be utilized most fully because in massive conductors the ponderomotive forces are not too critical as in thin bus-bar wires.

\section{CONCLUSION}

The electron scattering processes being rather complicated are conspicuous for samples of Corbino geometry so the approximation of relaxation time as a parameter independent on magnetic field is unadequated for describing of relaxation processes even at helium temperature.

Experimentally observed self magnetic field is nonuniform along sample radius, its magnitude correlates with estimations having been done on the base of presentation of Hall drift and peculiarities of electron relaxation processes. The self magnetic field coincides with an external one in direction providing current flows to the sample center.

The characteristic time of disk shaped sample as an inductive element have been calculated for different current and external field magnitudes. It is of the order of second for investigated here samples. The inductance and conductance differ about $40 \%$ for paramagnetic and diamagnetic current flow.

The estimated averaged energy density of self magnetic field is of the order of $10^{5} \mathrm{j} / \mathrm{m}^{3}$ that is of the same order of magnitude with the traditional inductive energy storage, an external magnetic field and the current flow through the sample being of $8 \mathrm{~T}$ and $10^{3} \mathrm{~A}$ respectively.

The experimental data and analysis show that the efficiency of disk shaped sample (for example an energy density of self magnetic field) as an inductor or inductive energy storage at hydrogen will be a third of that taking place at helium temperature.

\section{ACKNOWLEDGMENT}

This research is supported by Fund of Fundamental Investigations of the Republic of Belarus (Grant No. F40-249). The authors are very grateful to Belarusian Soros Foundation for a chance of presentation this work at CEC/ICMC'95 (Grant No. B9504-1025-33).

\section{REFERENCES}

1. D.A.Kleinman and A.L.Shawlow, Corbino disk, J.of Appl.Phys. 31:2176(1960),

2. G.P.Carver, A Corbino disk apparatus to measure hall mobilities in amorphous semiconductors, Rev.Sci.Instrum. 43:1257(1972),

3. E.K.Inall, A.E.Robson, and R.J.Turchi, Application of the Hall effect to the switching of inductive circuits, Rev.Sci. Instrum. 48:462(1977),

4. H.N.De Lang. H.van Kempen, and P.Wyder, The lattice thermal conductivity of very pure aluminium, J.Phys.F:Metal Phys. 8:L39(1978),

5. H.N.De Lang, H.van Kempen, and P.Wyder, Quadratic magnetoresistivity of closed orbit uncompensated metals, Phys.Rev.B. 20:809(1979),

6. J.A.M.M.van Haaren, G.J.C.L.Bruls, A.P.van Gelder, H.van Kempen, and P.Wyder, Thickness variations and the Corbino effect, Phys.Rev. 34:6813(1986),

7. B.B.Boiko, V.I.Gostishchev, A.A.Drozd, V.S.Kuzmin, and O.N.Mazurenko, Corbino effect in aluminum, Fiz. Met. i Metalloved. (Russian). 63:1133(1987). 